Journal of Computer Science 3 (12): 914-917, 2007

ISSN 1549-3636

(C) 2007 Science Publications

\title{
Black White Color and Gray Pixel Extraction Model
}

\author{
${ }^{1}$ K. Selvam and ${ }^{2}$ B. Poorna \\ ${ }^{1}$ Department of Computer Applications, \\ Dr. M.G.R. University, Chennai 600 095, India \\ ${ }^{2}$ Department of Computer Applications, \\ Easwari Engineering College, Chennai 600 089, India
}

\begin{abstract}
This is a design approach of software on image and pattern recognition, while eliminating the color and gray code of an image for comparison. This approach is quite effective for black and white and color images or scenes. Here we select particular part or parts of a given image and we compare with the contents of template-image (biometric) database. This approach will be the key idea for all the verifications and identification systems of Automated Biometric Analysis. Here the input is image (black and white or color) the output is a set of picture elements (pixels), we have various approaches they are (1) Minterm (minimized term) extraction for black and white image analysis and handling various gray levels through variables like A, B, C, D and the inverse (negative) image of the same with corresponding gray levels can also be maintained by their inverse like A', B',C',D'...respectively. (2) Maxterm (maximized term) the color images are generated by three primary colors (Red, Green, Blue) and this image can be recognized by both Minterm and Maxterm approaches and consider any one of the primary colors through Minterm approach and various gray levels of the primary color can be identified and analyzed by variables like A, B, C, D and the inverse (negative)image gray-levels can be performed by $\mathrm{A}^{\prime}, \mathrm{B}^{\prime}, \mathrm{C}^{\prime}, \mathrm{D}^{\prime}, \ldots$... respectively. Further the remaining two primary colors will be treated by the inverse of the earlier function of selected primary color (but not by the inverse variables) i.e., (A, B, C, D,) of primary color approach (maxterm approach).
\end{abstract}

Key words: Prime implicant, sum of products, gray, minterm, probabilistic determination

\section{INTRODUCTION}

Image or Pattern recognition is concerned with the automatic detection or classification of object or events. Pattern recognition is mostly used for the recognition of medical images, magnetic images, human speech, finger prints, hand shape and size, retinal scans, voice characteristics, typing pattern and hand writing, etc.,... $[1,2]$. Edge and line detection is another technique and feature on (i) Construction of image (ii) Image analysis iii) Extraction process with Geometric method or model and Mathematical (Boolean) approach. In earlier and existing method, pattern recognition is concerned by clustering process by probabilistic and deterministic method or modeling and function for pattern recognition is mostly as probabilistic determination $\sum f(x, y)$ i.e., fraction image $\sum \mathrm{f}(\mathrm{x}, \mathrm{y})=\left[\mathrm{g}_{\max }(\mathrm{x}, \mathrm{y})-\mathrm{g}_{\min }(\mathrm{x}, \mathrm{y})\right] / \mathrm{g}_{\max }(\mathrm{x}, \mathrm{y}) . \quad[3,5]$.

Thus the above said existing technique will give the result through approximation or probabilistic and deterministic method, this will be suitable for analogous process but not perfectly suitable for digital process and in digital analysis both analog and digital method are wealthy towards result. So each and every pixel is compared with the new extraction approach.

\section{PROBLEM DEFINITION}

An approach for the recognition of fingerprints of given part or whole of the image is done. The image is a set of pixels which is compared with the pixels of image stored in the database. The image before being stored in the database is subject to normalization, with the core point of the fingerprint as reference point. The image of the fingerprint to be matched is taken and its core point is identified. Around the core point a portion of the image is taken (say 500 pixels) in both the images and compared. The portion may be considered to be rectangular and can be divided into four quadrants. The image to be compared may have to be rotated $90^{\circ}, 180^{\circ}$ or $270^{\circ}$ for the quadrants to match. Once the images are normalized they can be compared using the Quine Mc Cluskey's method used for minimization of Boolean expressions.

Corresponding Author: K. Selvam, Department of Computer Applications, Dr.M.G.R. University, Chennai 600 095, India 


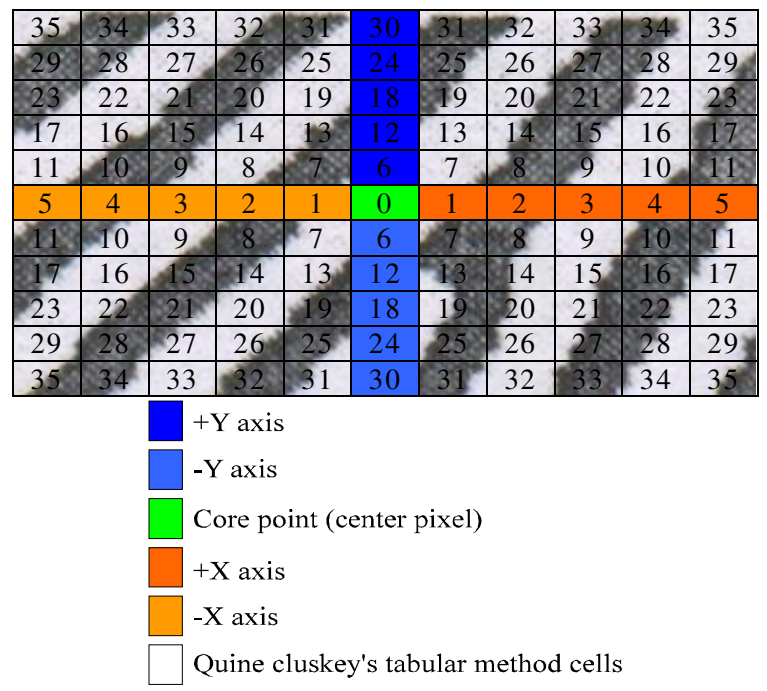

Fig. 1: Pixel pattern arrangement of biometric image

Whenever there is a gray value in a particular square of the image and it is treated as one (1), otherwise zero (0). By applying the minimization method a minimized Boolean expression is obtained for both the images. If the two expressions are the same then the inference is that they are similar. Also, when the image is distorted due to noise this method still works as it forms tow sets each for the image in the database and the image to be matched. One set has as its elements all the pixel positions which have a gray value called $\mathrm{X}$ and the other set $\mathrm{Y}$ has as its elements all the pixel positions that are free (Fig. 1).

The sets $\mathrm{X}$ and $\mathrm{Y}$ can be compared and a matching can be determined within allowable limits. This method can also be applied on two images one of which is the inverse of the other (negative image) as here the set $\mathrm{X}$ of one image will match with set $\mathrm{Y}$ of the other image and vice versa.

This method can be extended to non biometric images also when an appropriate normalization of images are done (Fig. 2).

i.e. Note that each Maxterm is the compliment of its corresponding minterm and vise-versa. A Boolean function may be expressed algebraically from a given Table 1 by forming a minterm for each combination of the variable which produces a 1 in the function and add of all the terms. eg. Function of three variables. [4,6,7].

The function 1 or $f_{R}$ in the above Table 2 , is determined by expressing the combination of 001,100 and 111 as x'y'z, xy'z',xyz respectively. Since the minterm result in $f_{1}=1$. i.e., Boolean function $f_{1}=x^{\prime} y^{\prime} z+x y^{\prime} z^{\prime}+x y z=m_{1}+m_{4}+m_{7}$. This is for one of

the primary colors (red) (Fig. 3).

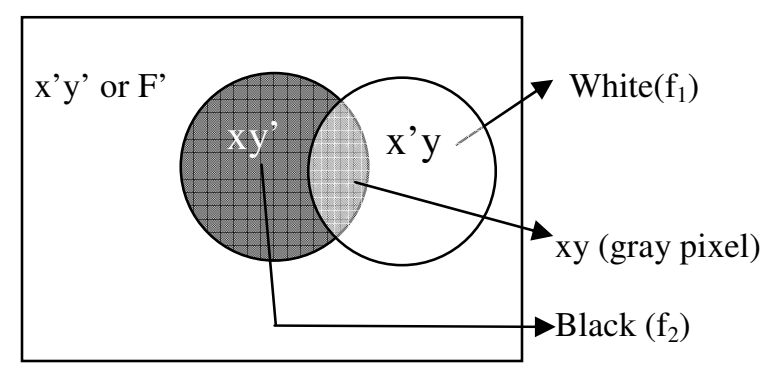

Fig. 2: Black and white pixel in an Image

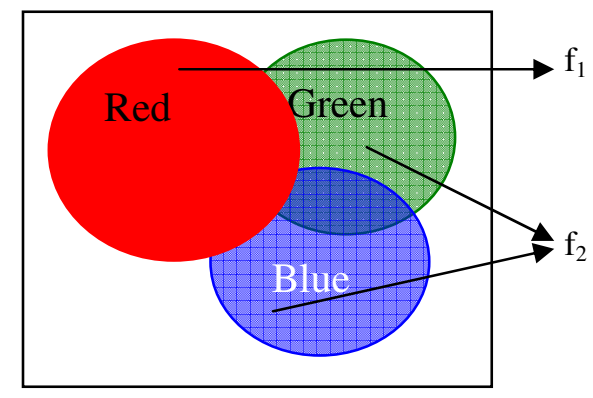

Fig. 3: Primary color pixels in an Image

\begin{tabular}{|c|c|c|c|c|c|c|}
\hline \multicolumn{2}{|c|}{ XYZ } & \multirow[b]{2}{*}{ Z } & \multicolumn{2}{|c|}{ Minterm } & \multicolumn{2}{|l|}{ Maxterm } \\
\hline X & Y & & Term & Designation & Term & Designation \\
\hline 0 & 0 & 0 & $X^{\prime} Y^{\prime} Z^{\prime}$ & $\mathrm{m}_{0}$ & $\mathrm{X}^{\prime}+\mathrm{Y}^{\prime}+\mathrm{Z}^{\prime}$ & $\mathrm{M}_{0}$ \\
\hline 0 & 0 & 1 & $X^{\prime} Y^{\prime} Z$ & $\mathrm{~m}_{1}$ & $X^{\prime}+Y^{\prime}+Z$ & $\mathrm{M}_{1}$ \\
\hline 0 & 1 & 0 & X'YZ' & $\mathrm{m}_{2}$ & $X^{\prime}+Y+Z^{\prime}$ & $\mathrm{M}_{2}$ \\
\hline 0 & 1 & 1 & X'Y Z & $\mathrm{m}_{3}$ & $X^{\prime}+Y+Z$ & $\mathrm{M}_{3}$ \\
\hline 1 & 0 & 0 & X Y'Z' & $\mathrm{m}_{4}$ & $X+Y^{\prime}+Z^{\prime}$ & $\mathrm{M}_{4}$ \\
\hline 1 & 0 & 1 & XY'Z & $\mathrm{m}_{5}$ & $X+Y^{\prime}+Z$ & $\mathrm{M}_{5}$ \\
\hline 1 & 1 & 0 & X Y Z' & $\mathrm{m}_{6}$ & $X+Y+Z^{\prime}$ & $\mathrm{M}_{6}$ \\
\hline 1 & 1 & 1 & X Y Z & $\mathrm{m}_{7}$ & $\mathrm{X}+\mathrm{Y}+\mathrm{Z}$ & $\mathrm{M}_{7}$ \\
\hline
\end{tabular}

Assume total (Black and White) function $F=\mathrm{f}_{1}+\mathrm{f}_{2}$

Table 2: Primary color pixel description

\begin{tabular}{llllll}
\hline $\mathrm{X}$ Y Z & & & & & \\
$-\mathrm{X}$ & $\mathrm{Y}$ & $\mathrm{Z}$ & $\begin{array}{l}\text { Function } 1 \\
\left(\mathrm{f}_{\mathrm{R}}\right)\end{array}$ & $\begin{array}{l}\text { Function } 2 \\
\left(\mathrm{f}_{\mathrm{G}}\right)\end{array}$ & $\begin{array}{l}\text { Function 3 } \\
\left(\mathrm{f}_{\mathrm{B}}\right)\end{array}$ \\
\hline 0 & 0 & 0 & 0 & 0 & 1 \\
0 & 0 & 1 & 1 & 0 & 1 \\
0 & 1 & 0 & 0 & 0 & 1 \\
0 & 1 & 1 & 0 & 1 & 0 \\
1 & 0 & 0 & 1 & 0 & 0 \\
1 & 0 & 1 & 0 & 1 & 0 \\
1 & 1 & 0 & 0 & 1 & 0 \\
1 & 1 & 1 & 1 & 1 & 0
\end{tabular}

Assume total color function $F=f_{R}+f_{G}+f_{B}$

This above example demonstrates an important property of Boolean Algebra, now consider the compliment of the Boolean function, it may be read from the Table 2 by forming a minterm for each combination that produces a 0 in the function and add of those terms. i.e., the compliment of $\mathrm{f}_{1}$ is read as $\mathrm{f}_{1}{ }^{\prime}=$ $x^{\prime} y^{\prime} z '+x$ 'yz'+x'yz+xy'z+xyz' this is data for the

where $I=$ Red or Green or Blue and corresponding gray-levels depending upon required color. 
Table 3: Black or color or gray pixel analysis

\begin{tabular}{|c|c|c|c|c|c|c|c|c|c|c|c|}
\hline \multirow[b]{2}{*}{ No of 1's } & \multirow{2}{*}{$\begin{array}{l}\text { Decimal } \\
\text { value }\end{array}$} & \multicolumn{4}{|c|}{ Minterms (on binary's) } & \multirow{2}{*}{$\begin{array}{l}\text { Check } \\
\text {...Ist }\end{array}$} & \multirow{2}{*}{$\begin{array}{l}\text { Prime } \\
\text { implicant } \\
\text { (PI) }\end{array}$} & \multirow{2}{*}{$\begin{array}{l}\text { First } \\
\text { stage of } \\
\text { elimination }\end{array}$} & \multirow{2}{*}{$\begin{array}{l}\text { Check } \\
\ldots .2 \text { nd }\end{array}$} & \multirow{2}{*}{$\begin{array}{l}\text { Prime } \\
\text { implicant } \\
\text { (PI) }\end{array}$} & \multirow{2}{*}{$\begin{array}{l}\text { Second } \\
\text { stage of } \\
\text { elimination }\end{array}$} \\
\hline & & $\mathrm{I}_{1}$ & $\mathrm{I}_{2}$ & $\mathrm{I}_{3}$ & $\mathrm{I}_{4}$ & & & & & & \\
\hline 0 & 0 & 0 & 0 & 0 & 0 & $*$ & 0,2 & $00 \times 0$ & $*$ & $0,2,8,10$ & X0 X0\# \\
\hline 1 & 2 & 0 & 0 & 1 & 0 & $*$ & 0,8 & X 0000 & $*$ & $0,8,2,10$ & X $0 \times 0 \#$ \\
\hline 1 & 8 & 1 & 0 & 0 & 0 & $*$ & 2,3 & $001 \mathrm{X}$ & $*$ & $2,3,10,11$ & X $01 \mathrm{X} \#$ \\
\hline 2 & 3 & 0 & 0 & 1 & 1 & $*$ & 2,10 & X 010 & $*$ & & \\
\hline 2 & 5 & 0 & 1 & 0 & 1 & $*$ & 8,10 & $10 \times 0$ & $*$ & & \\
\hline 2 & 10 & 1 & 0 & 1 & 0 & $*$ & 3,11 & X 011 & \# & & \\
\hline 3 & 11 & 1 & 0 & 1 & 1 & $*$ & 5,13 & X 101 & \# & & \\
\hline 3 & 13 & 1 & 1 & 0 & 1 & $*$ & 10,11 & $101 \mathrm{X}$ & $*$ & & \\
\hline
\end{tabular}

remaining two primary colors $\left(f_{G}+f_{B}\right)$. For other two primary colors the Boolean form can be obtained by $f_{1}$, $=(x+y+z) \cdot\left(x+y^{\prime}+z\right) \cdot\left(x+y^{\prime}+z^{\prime}\right) \cdot\left(x^{\prime}+y+z^{\prime}\right) \cdot\left(x^{\prime}+y^{\prime}+z\right)=$ $M_{0} \cdot M_{2} \cdot M_{3} \cdot M_{5} \cdot M_{6}$. It is possible to read the expression for individual color (primary).

\section{TREATMENT FOR PRIMARY COLOR RED}

The individual primary colors and their corresponding gray levels can be treated and maintained for Red color $R_{1}, R_{2}, R_{3}, R_{4}, \ldots$ and inverse $\mathrm{R}_{1}{ }^{\prime}, \mathrm{R}_{2}, \mathrm{R}_{3}{ }^{\prime}, \mathrm{R}_{4}$,,$\ldots$ i.e., add of the minterm and it consists the data of remaining two primary colors i.e. Blue and Green, But $\mathrm{R}_{1}, \mathrm{R}_{2}, \mathrm{R}_{3}, \mathrm{R}_{4}, \ldots$ are used for various gray levels of the primary red. This above method is efficiently performed by Quine Mc Cluskey's Tabular Method. Consider picture data (image) in the given form $\mathrm{f}(\mathrm{image})=\mathrm{f}\left(\right.$ gray $_{1}$, gray $_{2}$, gray $_{3}$, gray $\left._{4}\right)$, Example $\mathrm{f}\left(\mathrm{I}_{1}, \mathrm{I}_{2}, \mathrm{I}_{3}, \mathrm{I}_{4},\right)=\mathrm{f} \sum(0,2,3,5,8,10,11,13)$, for obtaining the simplified result(Boolean). This Boolean function will be the data of the same, further separate green and blue colors are also treated as the above treatment for red. but it is result of thinned and cleansed image, because here we select only the required primary color and corresponding gray-level pixels. Through this simplification method we do analyze both Black and White or Color images. If Primary color Red is performed by $f_{1}$, then remaining primary colors can be performed by $\mathrm{f}_{1}$ '. And here at the Table 3 a \# marked elements are considered for resultant. But * marked were compared and eliminated and shaded are Identical, so we take only one term.

\section{RESULT}

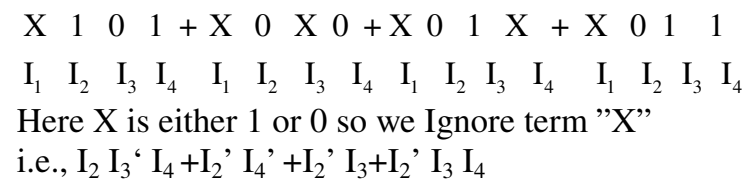

The result $=\mathrm{f}_{1}=\mathrm{I}_{2} \mathrm{I}_{3} \mathrm{I}_{4}+\mathrm{I}_{2}{ }^{\prime} \mathrm{I}_{4}{ }^{\prime}+\mathrm{I}_{2}{ }^{\prime} \mathrm{I}_{3}+\mathrm{I}_{2}{ }^{\prime} \mathrm{I}_{3} \mathrm{I}_{4}$

Example:

Assume Primary color Red $=f_{R}=f\left(R_{1}+R_{2}+R_{3}+R_{4}\right)$

Red- image data $=f_{R}=R_{2} R_{3} R_{4}+R_{2} R_{4}+R_{2} R_{3} R_{4}$ $=\mathrm{M}_{1}+\mathrm{M}_{4}+\mathrm{M}_{7}$

Similarly Primary Blue, Green are obtained by $f_{R}{ }^{\prime}=\left(f_{B}+f_{G}\right)$. Thus we do the same process for extracting all primary colors and this is apt to all the type of image and scenes, this is the distinct idea to approach the image extraction.

An attempt has been made to apply the existing method of minimization of Boolean expression to image processing and also it can be applied to any image with appropriate normalization procedures.

The research has been modified after including the necessary background information in the Introduction section. The appropriate details are included in the research and also in the reference section.

\section{CONCLUSIONS}

Now the image extraction can be handled for both Black and White, Color images, through this same approach and required gray level may be particularly performed and treated, individual primary color and corresponding gray levels can be analyzed. We can extract any of the primary colors to analyze the nature of the image and can analyze the brightness of the image also; this approach is effective for both image and inverse (negative) of the image also.

\section{REFERENCES}

1. Davide Maltoni, Davio Maie, A.K. Jain and Salil Prabakar, 2003. Hand Book of Fingerprint Recognition. Springer Professional Computing, pp: 176-213. 
2. Nalini Ratha and Ruud Bolle, 2003. Automatic Fingerprint Recognition System. Springer, pp: 67-112.

3. Jain, A.K., 1988. Fundamentals of Digital Image Processing. Prentice-Hall, Englewood Cliffs, NJ, pp: 36-74.

4. Moris Mano, 2002. Digital Computer Fundamentals. Prentice Hall, pp: 72-112.

5. Earl Gose, Richard Johnsonbaugh and SteveJost, 2000. Pattern recognition and Image Analysis, pp: 231-247.
6. Gonzalez, C. and R.E. Woods, 2002. Digital Image Processing (2nd Edn.), Prentice-Hall, Englewood Cliffs, NJ, pp: 36-113.

7. Rafael, C., Gonzalez and Richard E.Woods, 2002. Digital Image Processing, pp: 18-69. 